Pacific Journal of Mathematic 


\title{
AN OBSTRUCTION TO EXTENDING ISOTOPIES OF PIECEWISE LINEAR MANIFOLDS
}

\author{
EwING L. LusK
}

Let $F: M \times I^{n} \rightarrow Q \times I^{n}$ be an $n$-isotopy (not necessarily PL) of a compact PL $m$-manifold $M$ in a PL $q$-manifold $Q$, and let $G: Q \times I^{n} \rightarrow Q \times I^{n}$ be an ambient isotopy of $Q$ which covers $F$ on $Q \times \partial I^{n}$. If $m \leqq q-3$ there is in $\pi_{n} \operatorname{PL}(M, Q)$ an obstruction to finding an ambient isotopy of $Q$, isotopic to $G$, which covers $F$ and agrees with $G$ on $Q \times \partial I^{n}$.

1. Introduction. In the proof of the Hudson-Zeeman covering isotopy theorem [6], one has no control over the homeomorphism of the ambient manifold which one obtains at the end of the isotopy. In general, one might ask for sufficient conditions under which a given $n$-isotopy $F: M \times I^{n} \rightarrow Q \times I^{n}$ of one PL manifold in another, fixed on $\partial M$, can be covered by an ambient $n$-isotopy $H: Q \times I^{n} \rightarrow Q \times I^{n}$ fixed on $\partial Q$, in such a way that $H \mid Q \times \partial I^{n}$ is equal to some given levelpreserving homeomorphism $G$ of $Q \times \partial I^{n}$ which covers $F \mid M \times$ $\partial I^{n}$. Necessary conditions are that $F$ be level-preservingly locally flat and that $G$ have some extension to $Q \times I^{n}$ which is fixed on $\partial Q$. That these conditions are not sufficient can be seen by considering an isotopy $F: S^{1} \times I \rightarrow I^{2} \times I$ of a circle in the interior of $I^{2}$ which rotates the circle through $360^{\circ}$. Since $F$ can be chosen PL and locally flat, it follows from the ordinary covering isotopy theorem [6] that $F$ can be covered by an ambient isotopy $H$ of $I^{2}$ which is fixed on $\partial I^{2}$. But if $G: \partial\left(I^{2} \times I\right) \rightarrow \partial\left(I^{2} \times I\right)$ is the identity homeomorphism, then $H$ cannot be an extension of $G$. The difficulty here arises from the fact that the space of embeddings of $S^{1}$ into $I^{2}$ is not simply connected. The theorem below extends results of Gluck, Husch, and Rushing $[3,8]$. Let $M$ and $Q$ be PL $m$ - and $q$-manifolds respectively, with $M$ compact, and let $\operatorname{PL}(M, Q ; f)$ denote the semi-simplicial complex of proper PL embeddings of $M$ into $Q$, with base point $f$.

THEOREM 1. Let $F: M \times I^{n} \rightarrow Q \times I^{n}$ be a proper levelpreservingly locally flat $n$-isotopy (not necessarily $\mathrm{PL}$ ) fixed on $\partial M$. Let $G: Q \times I^{n} \rightarrow Q \times I^{n}$ be an ambient $n$-isotopy of $Q$, fixed on $\partial Q$, such that $G \circ\left(F_{0} \times 1\right)\left|M \times \partial I^{n}=F\right| M \times \partial I^{n}$. Suppose that $m \leqq$ $q-3$. Then there is a homeomorphism $h$ of $Q$ such that $h F_{0}$ is $P L$ and an obstruction $\alpha$ in $\pi_{n} \operatorname{PL}\left(M, Q ; h F_{0}\right)$ such that $\alpha=0$ if and only if there is a level-preserving isotopy $K$ of $Q \times I^{n}$, fixed on $\partial\left(Q \times I^{n}\right)$, such that $K_{1} G \circ\left(F_{0} \times 1\right)=F$; i.e. $K_{1} G$ extends $G \mid Q \times \partial I^{n}$ and covers $F$. 
REMARK 1. If $F$ and $G$ are PL, then the local flatness condition on $F$ need not be level-preserving, and $K$ can be taken to be PL. The proof of Theorem 1 in this PL case is like the proof given in [8] for the case $n=1$ and so is known. In the topological case, Theorem 1 follows straightforwardly from the fact that the inclusion $\operatorname{PL}(M, Q) \subset$ $\operatorname{TOP}(M, Q)$ is dense and a weak homotopy equivalence (See Theorem 2 below).

REMARK 2. Various combinations of dimension and connectivity conditions are sufficient to ensure that $\pi_{n} \operatorname{PL}\left(M, Q ; h F_{0}\right)=0$ and hence that the obstruction vanishes. We list some of them here. (See [7] and [9].)

(a) $\pi_{r}(Q)=0$ for $n \leqq r \leqq m+n$ and $2 m+n \leqq q-2$.

(b) $M$ is $(2 m-q+n)$-connected, $Q$ is $(2 m-q+n+1)-$ connected, $\pi_{r}(Q)=0$ for $n \leqq r \leqq m+n$, and $m+n \leqq q-2$.

(c) $\pi_{r}(Q)=0$ for $n \leqq r \leqq m+n, \quad F_{0}$ is $(2 m-q+n+1)-$ connected, and $m+n \leqq q-2$.

2. Definitions. Let $I^{n}$ be the $n$-fold product of the unit interval $[0,1]$. The point $(0,0, \cdots 0)$ in $I^{n}$ will be denoted by 0 , and the subset $I^{n-1} \times 0 \cup \partial I^{n-1} \times I$ of $I^{n}=I^{n-1} \times I$ will be denoted by $J^{n-1}$. An $n$-isotopy of $M$ in $Q$ is an embedding $F: M \times I^{n} \rightarrow Q \times I^{n}$ which is level-preserving ( $p \circ F=p$ where $p$ is projection onto $I^{n}$ ). It is proper if $F^{-1}\left(\partial Q \times I^{n}\right)=\partial M \times I^{n}$. An embedding $F_{t}: M \rightarrow Q$ is defined for each $t \in I^{n}$ by $F(x, t)=\left(F_{t}(x), t\right)$. A 1-isotopy is called an isotopy, and $F_{0}$ and $F_{1}$ are said to be isotopic. An $n$-isotopy $F$ is fixed on $X$ if $F\left|X \times I^{n}=F_{0} \times 1\right| X \times I^{n}$, where 1 denotes the identity map. It is level-preservingly locally flat if for each $(x, t) \in M \times I^{n}$ there is a neighborhood $N$ of $t$ in $I^{n}$, a level-preserving embedding $H$ of either $E^{m} \times N$ or $E_{+}^{m} \times N$ into $M \times N$ (depending on whether $x$ is in int $M$ or $\partial M)$ with $H(0, t)=(x, t)$, and a level preserving embedding $G$ of either $E^{q} \times N$ or $E_{+}^{q} \times N$ into $Q \times N$ depending on whether $F_{t}(x)$ is in int $Q$ or $\partial Q$ ) with $G(0, t)=F(x, t)$, such that $G^{-1} F H$ is of the form $i \times 1$, where $i$ is the natural inclusion of $E^{m}$ into $E^{q}$ or $E_{+}^{m}$ into $E_{+}^{q}$, as the case may be. An ambient $n$-isotopy of $Q$ is a level-preserving homeomorphism $H$ of $Q \times I^{n}$ such that $H_{0}=1$. If $A \subset X$, an $\varepsilon$-push of $(X, A)$ is an ambient isotopy of $X$ which is fixed outside an $\varepsilon$ neighborhood of $A$.

We make use of the semi-simplicial complexes $\operatorname{Aut}_{\mathrm{PL}}(Q)$ and $\operatorname{PL}(M, Q)$, whose $k$-simplices are ambient $k$-isotopies of $Q$ fixed on $\partial Q$ and proper $k$-isotopies of $M$ in $Q$ fixed on $\partial M$, respectively. The Hudson covering $n$-isotopy theorem [5] can be used to prove, as in [4], that if $f: M \rightarrow Q$ is a given PL embedding then the simplicial map $p: \operatorname{Aut}_{\mathrm{PL}}(Q) \rightarrow \operatorname{PL}(M, Q)$ given by $p(H)=H \circ(f \times 1)$ is a fibration, i.e., 
given level-preserving embeddings $K: Q \times J^{n-1} \rightarrow Q \times J^{n-1}$ and $L: M \times$ $I^{n} \rightarrow Q \times I^{n}$ such that $p(K)=L \mid M \times J^{n-1}$, there is an $n$-isotropy $H: Q \times I^{n} \rightarrow Q \times I^{n}$ such that $p(H)=L$ and $H \mid Q \times J^{n-1}=K$. An element of $\pi_{n} \operatorname{PL}(M, Q ; f)$ is represented by a level-preserving PL embedding $L: M \times \partial I^{n+1}$ such that $L_{0}=f$.

3. Spaces of embeddings. In this section we consider the relationship between $\operatorname{PL}(M, Q)$ and $\operatorname{TOP}(M, Q)$, the semi-simplicial complex of topological embeddings of $M$ into $Q$. Recent work of Edwards and Miller $[2,12]$ has relaxed the dimension restrictions on the results in [10]. The key lemma is the following.

Lemma 1. Let $H: M \times I^{n} \rightarrow Q \times I^{n}$ be a level-preserving embedding. Suppose that $m \leqq q-3$ and $q \geqq 5$. Then for any $\varepsilon>0$ there is $a \delta=\delta(\varepsilon, H)>0$ such that if $G_{0}, G_{1}: M \times I^{n} \rightarrow Q \times I^{n}$ are level-preserving PL embeddings with $d\left(G_{i}, H\right)<\delta$, then there is a level-preserving $\varepsilon$-push $K$ of $\left(Q \times I^{n}, H\left(M \times I^{n}\right)\right)$ such that $K_{1} G_{0}=$ $G_{1}$. If $G_{0}$ and $G_{1}$ agree on $M \times \partial I^{n}$, then $K$ can be assumed fixed on $Q \times \partial I^{n}$.

Proof. If $H$ is of the form $h \times 1$ for some embedding $h: M \rightarrow Q$, then the lemma follows directly from Corollary 2 of [2] and Corollary 3 of [1]. Generalization to the case in which $H$ is not of this form can be carried out as in the second half of the proof of Theorem $4.2(\mathrm{~m}, \mathrm{~s})$ in [10].

REMARK 3. The above "local solvability" result is the basis for Theorems 2.1-2.5 of [10] which are stated there with more stringent dimension restrictions. We may now regard those results to be true for $m \leqq q-3, q \geqq 5$. In particular, Theorems 2.1 and 2.4 give us

THEOREM 2. If $m \leqq q-3$ and $q \geqq 5$, then the inclusion $\operatorname{PL}(M, Q) \subset \operatorname{TOP}(M, Q)$ is dense and a weak homotopy equivalence; i.e., if $f: M \rightarrow Q$ is $\mathrm{PL}$, then the homomorphism $i_{*}: \pi_{n} \operatorname{PL}(M, Q ; f) \rightarrow$ $\pi_{n} \operatorname{TOP}(M, Q ; f)$ induced by inclusion is an isomorphism for all $n$.

4. Proof of Theorem 1. The following lemma, which is Theorem 2.3 of [10] with the new dimension conditions, makes possible the treatment of the non-PL case with PL techniques.

Lemma 2. Let $F: M \times I^{n} \rightarrow Q \times I^{n}$ be a level-preservingly locally flat proper $n$-isotopy which is PL on $\partial\left(M \times I^{n}\right)$. Suppose $m \leqq q-3$ and $q \geqq 5$, and that $\varepsilon>0$ is given. Then there is a level-preserving 
$\varepsilon$-push $T$ of $\left(Q \times I^{n}, F\left(M \times I^{n}\right)\right)$, fixed on $\partial\left(Q \times I^{n}\right)$, such that $T_{1} F$ is PL.

Proof of Theorem 1. By Lemma 2 with $n=0$ (See [11]), there is a small homeomorphism $h$ of $Q$ such that $h F_{0}: M \rightarrow Q$ is PL. Consider the embedding $(h \times 1) G^{-1} F: M \times I^{n} \rightarrow Q \times I^{n}$. Since it is a levelpreservingly locally flat $n$-isotopy and $(h \times 1) G^{-1} F \mid \partial\left(M \times I^{n}\right)=$ $\left(h F_{0}\right) \times 1$, which is PL, there is by Lemma 2 a level-preserving isotopy $T$ of $Q \times I^{n}$, fixed on $\partial\left(Q \times I^{n}\right)$, such that $T_{1}(h \times 1) G^{-1} F$ is PL. Now define $L: M \times \partial I^{n+1} \rightarrow Q \times \partial I^{n+1}$ by considering $I^{n+1}$ as $I^{n} \times I$ and letting $L$ be $T_{1}(h \times 1) G^{-1} F$ on $M \times I^{n} \times 1$ and $\left(h F_{0}\right) \times 1$ on $M \times J^{n}$. Then $L$ is PL and so represents an element $\alpha$ of $\pi_{n} \operatorname{PL}\left(M, Q ; h F_{0}\right)$. To say $\alpha=0$ in $\pi_{n} \operatorname{PL}\left(M, Q ; h F_{0}\right)$ is to say that there is a PL $(n+1)$-isotopy $H^{\prime}: M \times I^{n+1} \rightarrow Q \times I^{n+1}$ such that $H^{\prime} \mid M \times \partial I^{n+1}=L$. Therefore we can use the lifting property of the fibration $p: \operatorname{Aut}_{\mathrm{PL}}(Q) \rightarrow \operatorname{PL}(M, Q)$ given by $p(K)=K \circ\left(h F_{0} \times 1\right)$ to find an ambient $(n+1)$-isotopy $H^{\prime \prime}: Q \times I^{n+1} \rightarrow Q \times I^{n+1}$ such that $H^{\prime \prime} \mid Q \times J^{n}=1$ and $H^{\prime \prime} \circ\left(h F_{0} \times 1\right)=$ $H^{\prime}$. Now we define

$$
\begin{aligned}
K= & (G \times 1)\left(h^{-1} \times 1 \times 1\right) T^{-1} H^{\prime \prime}(h \times 1 \times 1)\left(G^{-1} \times 1\right): \\
& \left(Q \times I^{n}\right) \times I \rightarrow\left(Q \times I^{n}\right) \times 1 .
\end{aligned}
$$

Then $K_{1} G$ covers $F$ and extends $G \mid Q \times I^{n}$, as desired.

Conversely, if $K$ exists with the desired properties, then $K^{\prime}: M \times$ $\partial I^{n+1} \times I \rightarrow Q \times \partial I^{n+1} \times I$ defined by $K_{t}^{\prime}=T_{1-t}(h \times 1) G^{\prime} K_{1-t} G\left(F_{0} \times 1\right)$ on $M \times J^{n} \times I$ and $h F_{0} \times 1$ on $M \times\left(I^{n-1} \times 1\right) \times I$ is a level-preserving isotopy taking $L$ to $h F_{0} \times 1$. Therefore $\alpha$ is trivial as an element of $\pi_{n} \operatorname{TOP}\left(M, Q ; h F_{0}\right)$, the semi-simplicial complex of embeddings of $M$ into $Q$. By Theorem 2, $\alpha$ is trivial in $\pi_{n} \operatorname{PL}\left(M, Q: h F_{0}\right)$.

5. The obstruction $\alpha$. In the construction above, $\alpha$ appeared to depend on $h, T$, and $G$. In this section we show that $\alpha$ can be chosen in such a way that it depends only on $F$.

In applying Lemma 2 to construct $h$, above, we may choose $h$ so that $h F_{0}$ is within $\delta\left(F_{0}, 1\right)$ of $F_{0}$, where $\delta$ comes from Lemma 1. Any two such homeomorphisms $h$ and $h^{\prime}$ will then be such that $h F_{0}$ and $h^{\prime} F_{0}$ are ambient isotopic. Similarly we choose $T$ to be a $\delta\left((h \times 1) G^{-1} F, 1\right)$ push, so that if $T^{\prime}$ is another push which takes $(h \times 1) G^{-1} F$ to a $\mathrm{Pi}$ embedding, $T_{1}(h \times 1) G^{-1} F$ and $T_{1}^{\prime}(h \times 1) G^{-1} F$ are PL ambient isotopic, and the $\alpha$ 's constructed with them will be homotopic in $\pi_{n} \operatorname{PL}\left(M, Q ; h F_{0}\right)$.

Now suppose that $G$ and $G^{\prime}$ are level-preserving homeomorphisms of $Q \times I^{n}$ satisfying the hypotheses of the theorem. Since $G^{-1} F$ and 
$G^{\prime-1} F$ are each isotopic to $F_{0} \times 1$, they are isotopic. If we denote by $\alpha$ and $\alpha^{\prime}$ the obstructions constructed as above from $G$ and $G^{\prime}$, the isotopy of $G^{-1} F$ to $G^{\prime-1} F$ will induce a homotopy from $\alpha$ to $\alpha^{\prime}$ in $\pi_{n} \operatorname{TOP}(M, Q)$. By Theorem 2, $\alpha$ is homotopic to $\alpha^{\prime}$ in $\pi_{n} \operatorname{PL}\left(M, Q ; h F_{0}\right)$, and so $\alpha$ does not depend on $G$.

\section{REFERENCES}

1. R. D. Edwards, The equivalence of close piecewise linear embeddings, preprint.

2. R. D. Edwards, and R. T. Miller, Local connectivity of spaces of embeddings, Notices Amer. Math. Soc., 19 (1972), A-467.

3. H. Gluck, Restriction of isotopies, Bull. Amer. Math. Soc., 69 (1963), 78-82.

4. A. Haefliger, and V. Poenaru, Le classification des immersions combinatoires, Pub. Math. I.H.E.S. Paris, 23 (1964), 75-91.

5. J. F. P. Hudson, Extending piecewisise linear isotopies, Proc. London Math. Soc., 3, 16 (1966), $651-668$.

6. J. F. P. Hudson, and E. C. Zeeman, On combinatorial isotopy, Pub. Math. I.H.E.S., Paris, 19 (1964), 69-94.

7. L. S. Husch, Homotopy groups of PL embedding spaces, Pacific J. Math., 33 (1970), 149-155.

8. L. S. Husch, and T. B. Rushing, Restrictions of isotopies and concordances, Michigan Math. J., 16 (1966), 303-307.

9. E. L. Lusk, Homotopy groups of spaces of embeddings, Ph. D. thesis, University of Maryland, (1970).

10. Level-preserving approximations and isotopies, and homotopy groups of spaces of embeddings, Illinois J. Math., 18 (1974), 147-159.

11. R. T. Miller, Close isotopies on piecewise linear manifolds, Trans. Amer. Math. Soc., 151 (1970), 597-628.

12. - Fiber-preserving equivalence, preprint.

Received February 1, 1973.

NORTHERN ILLINOIS UNIVERSITY 



\section{PACIFIC JOURNAL OF MATHEMATICS \\ EDITORS}

\author{
Richard ARENS (Managing Editor) \\ University of California \\ Los Angeles, California 90024
}

\author{
R. A. Beaumont \\ University of Washington \\ Seattle, Washington 98105
}

\section{J. DugundJI}

Department of Mathematics

University of Southern California

Los Angeles, California 90007

D. Gilbarg and J. Milgram

Stanford University

Stanford, California 94305

\section{ASSOCIATE EDITORS}
E. F. BECKENBACH
B. H. NeumanN
F. WOLF
K. YoshidA

\section{SUPPORTING INSTITUTIONS}

\author{
UNIVERSITY OF BRITISH COLUMBIA \\ CALIFORNIA INSTITUTE OF TECHNOLOGY \\ UNIVERSITY OF CALIFORNIA \\ MONTANA STATE UNIVERSITY \\ UNIVERSITY OF NEVADA \\ NEW MEXICO STATE UNIVERSITY \\ OREGON STATE UNIVERSITY \\ UNIVERSITY OF OREGON \\ OSAKA UNIVERSITY
}

\author{
UNIVERSITY OF SOUTHERN CALIFORNIA \\ STANFORD UNIVERSITY \\ UNIVERSITY OF TOKYO \\ UNIVERSITY OF UTAH \\ WASHINGTON STATE UNIVERSITY \\ UNIVERSITY OF WASHINGTON \\ AMERICAN MATHEMATICAL SOCIETY
}

The Supporting Institutions listed above contribute to the cost of publication of this Journal, but they are not owners or publishers and have no responsibility for its contents or policies.

Mathematical papers intended for publication in the Pacific Journal of Mathematics should be in typed form or offset-reproduced (not dittoed), double spaced with large margins. Underline Greek letters in red, German in green, and script in blue. The first paragraph or two must be capable of being used separately as a synopsis of the entire paper. Items of the bibliography should not be cited there unless absolutely necessary, in which case they must be identified by author and Journal, rather than by item number. Manuscripts, in duplicate, may be sent to any one of the four editors. Please classify according to the scheme of Math. Reviews, Index to Vol. 39. All other communications should be addressed to the managing editor, or Elaine Barth, University of California, Los Angeles, California, 90024.

100 reprints are provided free for each article, only if page charges have been substantially paid. Additional copies may be obtained at cost in multiples of 50 .

The Pacific Journal of Mathematics is issued monthly as of January 1966. Regular subscription rate: $\$ 72.00$ a year (6 Vols., 12 issues). Special rate: $\$ 36.00$ a year to individual members of supporting institutions.

Subscriptions, orders for back numbers, and changes of address should be sent to Pacific Journal of Mathematics, 103 Highland Boulevard, Berkeley, California, 94708.

PUBLISHED BY PACIFIC JOURNAL OF MATHEMATICS, A NON-PROFIT CORPORATION Printed at Jerusalem Academic Press, POB 2390, Jerusalem, Israel.

$$
\begin{gathered}
\text { Copyright (c) } 1975 \text { Pacific Journal of Mathematics } \\
\text { All Rights Reserved }
\end{gathered}
$$




\section{Pacific Journal of Mathematics}

Vol. 56, No. 2 December, 1975

Ralph Alexander, Generalized sums of distances .................. 297

Zvi Arad and George Isaac Glauberman, A characteristic subgroup of a group of odd order ............................... 305

B. Aupetit, Continuité du spectre dans les algèbres de Banach avec involution ........................................ 321

Roger W. Barnard and John Lawson Lewis, Coefficient bounds for some classes of starlike functions . ...........................

Roger W. Barnard and John Lawson Lewis, Subordination theorems for some classes of starlike fumctions . .................... 333

Ladislav Bican, Preradicals and injectivity .................. 367

James Donnell Buckholtz and Ken Shaw, Series expansions of analytic

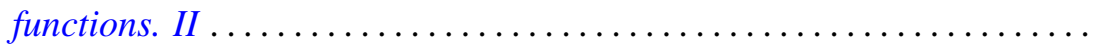

Richard D. Carmichael and E. O. Milton, Distributional boundary values in

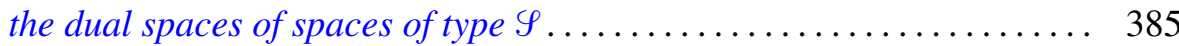

Edwin Duda, Weak-unicoherence ............................ 423

Albert Edrei, The Padé table of functions having a finite number of essential singularities ........................................

Joel N. Franklin and Solomon Wolf Golomb, A function-theoretic approach to the study of nonlinear recurring sequences ................ 455

George Isaac Glauberman, On Burnside's other $p^{a} q^{b}$ theorem 469

Arthur D. Grainger, Invariant subspaces of compact operators on topological vector spaces .........................

Jon Craig Helton, Mutual existence of sum and product integrals .

Franklin Takashi Iha, On boundary functionals and operators with finite-dimensional null spaces

Gerald J. Janusz, Generators for the Schur group of local and global number fields

A. Katsaras and Dar-Biau Liu, Integral representations of weakly compact operators.

W. J. Kim, On the first and the second conjugate points .

Charles Philip Lanski, Regularity and quotients in rings with involution ....

Ewing L. Lusk, An obstruction to extending isotopies of piecewise linear manifolds.

Saburou Saitoh, On some completenesses of the Bergman kernel and the Rudin kernel..

Stephen Jeffrey Willson, The converse to the Smith theorem for $Z_{p}$-homology spheres. 